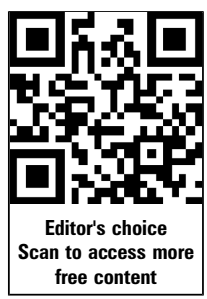

${ }^{1}$ Department of Pediatrics, Washington University in St. Louis, St. Louis, Missouri, USA

${ }^{2}$ Department of Paediatrics and Child Health, University of Malawi, Blantyre, Malawi ${ }^{3}$ Department of Community Health, University of Malawi, Blantyre, Malawi

${ }^{4}$ Children's Nutrition Research Center, Baylor College of Medicine, Houston, USA

\section{Correspondence to} Professor Mark Manary, Department of Pediatrics, One Children's Place, Campus Box 8116, St. Louis, MO 63110, USA; manary@wustl.edu

Received 31 July 2014 Revised 30 October 2014 Accepted 31 October 2014 Published Online First 24 November 2014

\section{CrossMark}

To cite: Trehan I, Manary MJ. Arch Dis Child 2015:100:283-287.

\title{
Management of severe acute malnutrition in low-income and middle-income countries
}

\author{
Indi Trehan, 1,2 Mark J Manary 1,3,4
}

\begin{abstract}
Kwashiorkor and marasmus, collectively termed severe acute malnutrition (SAM), account for at least $10 \%$ of all deaths among children under 5 years of age worldwide, virtually all of them in low-income and middle-income countries. A number of risk factors, including seasonal food insecurity, environmental enteropathy, poor complementary feeding practices, and chronic and acute infections, contribute to the development of SAM. Careful anthropometry is key to making an accurate diagnosis of SAM and can be performed by village health workers or even laypeople in rural areas. The majority of children can be treated at home with ready-to-use therapeutic food under the community-based management of acute malnutrition model with recovery rates of approximately $90 \%$ under optimal conditions. A small percentage of children, often those with HIV, tuberculosis or other comorbidities, will still require inpatient therapy using fortified milk-based foods.
\end{abstract}

\section{CLINICAL MANIFESTATIONS AND DIAGNOSIS}

Severe acute malnutrition (SAM) in children presents in at least three different forms in low-income and middle-income countries (LMICs). Careful, precise anthropometry is essential to accurately diagnose and manage children with SAM. This can be challenging in settings where large numbers of children are being screened in rapid fashion in rural health centres and requires an investment in the training of anthropometrists, provision of reliable and calibrated instruments, and quality control. ${ }^{12}$ Children should have their length measured to at least a precision of $0.5 \mathrm{~cm}$, weight to the nearest $100 \mathrm{~g}$ or less and mid-upper arm circumference (MUAC) to the nearest $2 \mathrm{~mm}$ or less. In practice, length can ideally be measured to the nearest $0.1 \mathrm{~cm}$ using rigid height boards, weight can be measured to the nearest $5 \mathrm{~g}$ using electronic scales powered by disposable batteries and MUAC to the nearest $1 \mathrm{~mm}$ using standard paper insertion tapes. Pitting oedema should be assessed and quantified in the feet, lower legs, hands and face as well. ${ }^{3}$

Children with acute wasting - mild, moderate or severe ('marasmus') - are often weak and emaciated, having suffered a significant amount of weight loss in a relatively brief period prior to presenting for care. Much of the weight loss is initially visible in the groin and axilla, and later in the buttocks, thighs and face. Children with marasmus are often considered to have an 'old man' appearance and are often either quite apathetic or inconsolable. Objective, reproducible anthropometric criteria should be used to make the diagnosis of marasmus rather than subjective criteria such as 'visible severe wasting. ${ }^{4}$ Marasmus is diagnosed based on either a MUAC $<115 \mathrm{~mm}$ or a weight-for-height $\mathrm{Z}$-score (WHZ) more than three SDs below the mean, ${ }^{5}$ based on the 2006 WHO growth standards. ${ }^{6}$

When resources are available, it is preferable to assess both MUAC and WHZ to identify children with marasmus as the two populations of children identified by these individual criteria will not overlap uniformly. In most populations, the children identified as severely wasted by WHZ will generally be older and at lower risk of death than those identified by MUAC. ${ }^{7}$ Colour-coded MUAC tapes requiring no literacy may also be distributed to laypeople to increase screening coverage in rural villages without the need for as many trained health workers. For these reasons, in contexts with limited human or material resources where only one screening method is available, it is preferable to screen by MUAC rather than WHZ. ${ }^{8}$ While anthropometry alone may classify children as marasmic and prompt therapy, underlying illnesses and triggers such as severe dehydration, malabsorption due to geohelminths, HIV-associated wasting and congenital anomalies should be considered while nutritional rehabilitation is undertaken.

A second form of SAM, kwashiorkor, is diagnosed based upon the cardinal physical finding of symmetric bilateral pitting oedema that begins in the feet (labelled $1+$ oedema, figure $1 \mathrm{~A}$ ), progresses to involve the lower legs and hands $(2+$ oedema, figure 1B) and in severe cases can involve the face (3 + oedema, figure 1C). Kwashiorkor was originally described among children who were rapidly weaned onto low-protein complementary foods from breast milk, and thus the pathophysiology was thought to be primarily one of protein deficiency, but further experience has questioned this hypothesis. ${ }^{9}$ 10 Children with kwashiorkor often have 'flaky paint' depigmentation of their skin, commonly leading to frank breakdown, which can serve as a portal of entry for infectious pathogens. ${ }^{11}$ The acute development of oedema among impoverished children in LMICs, even in children who have previously been growing well, is highly specific for kwashiorkor, although other diagnoses should of course still be kept under consideration, especially among those who do not recover as might be expected.

The third major form of SAM among children in LMICs, marasmic kwashiorkor, presents with both the severe wasting that characterises marasmus and the oedema that characterises kwashiorkor. These children are generally the most ill with the highest risk of mortality. ${ }^{12}$ The varying routes to these different forms of SAM among children with similar diets, genetics and environments have not been clearly elucidated, but the role of each individual's 
Figure 1 Kwashiorkor with progressive pitting oedema and cutaneous findings. (A) Bilateral pitting oedema of the feet represents $1+$ oedema in the context of kwashiorkor. (B) Progression of oedema to the arms is labelled as 2+ oedema.

(C) Progression of oedema to the face is classified as 3+ oedema.
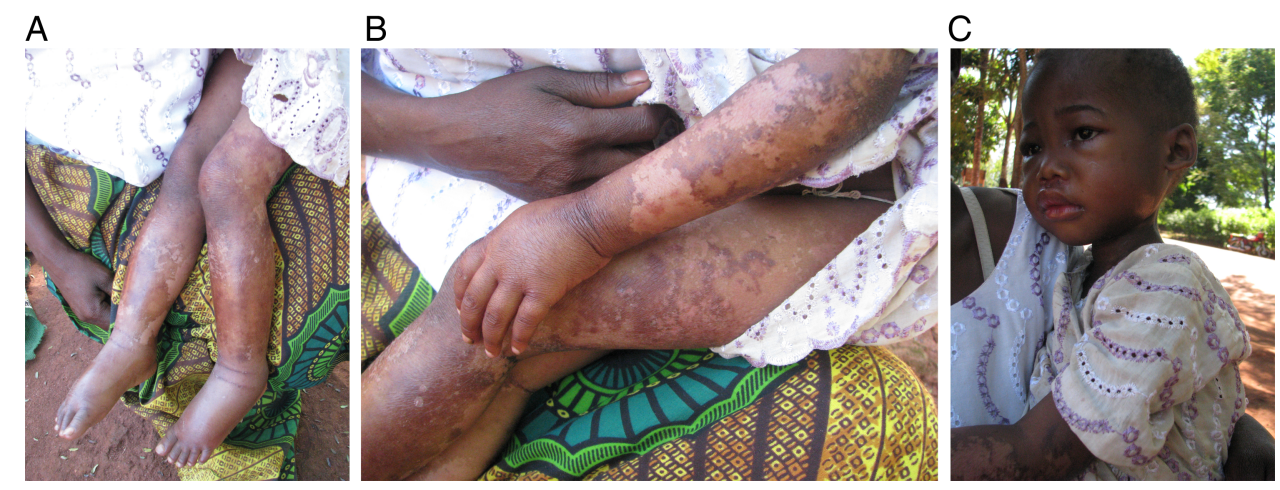

intestinal microbiome has gained recent attention as a plausible mechanism. ${ }^{13} 14$

Malnourished children often have a concomitant acute or chronic illness at the time of diagnosis, and similarly many children who present for medical care due to an acute illness are often found to be malnourished, unbeknown to their caretakers. The identification and diagnosis of malnourished children should thus ideally occur as part of an integrated health system rather than as a stand-alone service for acute malnutrition alone. An assessment for acute malnutrition, to include at least measuring MUAC and assessing for kwashiorkor, should occur as part of the general medical assessment of all children presenting for care in LMICs. In hospital settings, this should be part of the Emergency Triage Assessment and Treatment system, ${ }^{15}$ as the diagnosis of SAM will affect the therapies (such as the amount and speed of fluid resuscitation) given for the primary presenting symptom. In outpatient settings, similar assessments for acute malnutrition should occur as part of the Integrated Community Case Management framework. ${ }^{16}$

\section{EPIDEMIOLOGY AND IMPACT}

More than 500000 deaths each year among children under 5 years of age are attributable to marasmus, using the $\mathrm{WHZ}<-3$ definition, accounting for $>7 \%$ of all mortality in this age group. ${ }^{17}$ At any given time, some 18 million children in lowincome and middle-income countries are estimated to suffer from marasmus, ${ }^{17}$ most of them in Asia. ${ }^{18}$ However, this is likely a vast underestimate as it does not take into account children aged 6-59 months with MUAC $<115 \mathrm{~mm}$, nor has the incidence or prevalence of kwashiorkor been accurately quantified. ${ }^{19}$ These estimates also generally do not include acute man-made and natural humanitarian crises, during which large numbers of cases of SAM are also seen. ${ }^{20}$ Deservedly, concerted global efforts to address SAM in a cost-effective manner are warranted as reduction of mortality from this disease will contribute markedly to an overall global decrease in child mortality. ${ }^{21-23}$

The proportion of SAM due to kwashiorkor also varies widely by geography, with very high rates in southern Africa and lower rates in other parts of Africa, Latin America and Asia. Our own experience in West Africa suggests that approximately one-quarter of SAM cases there are due to kwashiorkor while in southern Africa approximately two-thirds to three-fourths of the cases of SAM we treat are due to kwashiorkor. ${ }^{12}$ All in all, it seems quite likely that the under-five mortality attributable to SAM exceeds $10 \%$.

What remains incompletely understood and yet of growing importance is the impact of episodes of SAM on a growing child's risk for metabolic syndrome and obesity in adulthood, ${ }^{24}$ particularly given the increasing recognition of a 'double burden' of overnutrition in adults superimposed on the endemic crisis of childhood undernutrition in many LMICs. ${ }^{17} 25$

\section{TREATMENT}

After being diagnosed with SAM, each child must be assessed for the presence of clinical complications that would warrant inpatient therapy (figure 2). ${ }^{26}$ The essential complication to assess for carefully is anorexia; all children with SAM should thus undergo a supervised test feeding of approximately $30 \mathrm{~g}$ of ready-to-use therapeutic food (RUTF). Other common complications include severe dehydration, high fever, respiratory distress, hypoglycaemia with lethargy and evidence of severe anaemia that may require blood transfusion. The clinical assessment for these complications can often-but not always-be streamlined to the appetite test as it is likely that most of these various significant complications will lead to the anorexia that would make a child ineligible for outpatient therapy. Children with SAM, due to chronic medical conditions such as congenital heart disease, cerebral palsy and other syndromes, may not 'pass' the appetite test and the therapies described herein for SAM may not be sufficient for them to achieve standard anthropometric goals. Directed therapies for their underlying illnesses should be provided whenever possible and the definition of nutritional recovery should be individualised in these cases as prolonged nutritional supplementation or enrolment in a therapeutic feeding programme may be futile.

Provider-initiated HIV testing and counselling is an important component of the initial management of all children with SAM in high-prevalence areas and can often reveal previously unsuspected cases of HIV in children and their mothers. Children with HIV who are otherwise well-appearing and demonstrate an appetite can still be treated as outpatients, ${ }^{27}$ although evidence suggests that early, possibly simultaneous, initiation of antiretroviral therapy in these cases is beneficial. ${ }^{28}$

In areas where active surveillance and case-finding for SAM is being conducted effectively, the vast majority of children with SAM will be identified early in their illness and not have any of these complications and will be able to enter into a feeding programme under the community-based management of acute malnutrition (CMAM) model. This treatment protocol is built upon RUTF as the key element in nutritional rehabilitation. ${ }^{29}$ While the most common recipe for RUTF consists of a peanut paste fortified with macronutrients and micronutrients to meet minimum nutrient specifications and quality standards established by the WHO (table 1$){ }^{30}$ novel formulations using locally sourced ingredients are being increasingly studied and developed in a number of settings. ${ }^{31}$ Ideally, RUTF will have a very low moisture content in order to minimise the risk of microbial contamination. Children who successfully complete a test feeding 


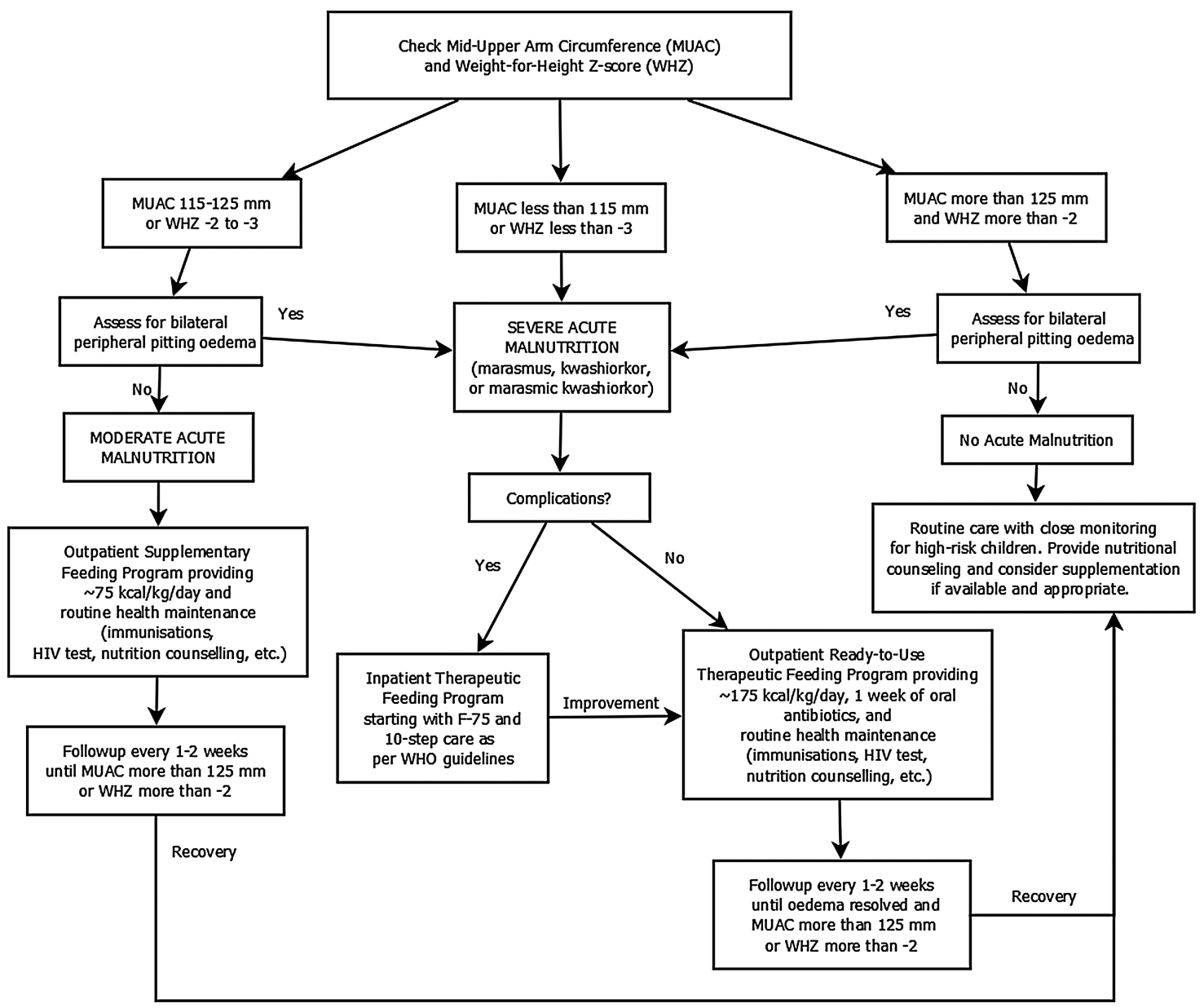

Figure 2 Diagnostic and treatment algorithm for acute malnutrition in children. Details of measurement of anthropometry in text and from 2009 WHO/Unicef guideline. ${ }^{5}$ Details of moderate malnutrition therapy are outlined elsewhere. ${ }^{41}$ Details of inpatient 10 -step therapy are given in figure 3 and in 2003 WHO guideline. ${ }^{26}$ Details of management of severe acute malnutrition are given in the text and in 2007 and 2013 WHO guidelines. ${ }^{30} 32$

under direct observation by trained staff can generally be discharged home with a 1-week to 2-week supply of RUTF at a dose of approximately $150-200 \mathrm{kcal} / \mathrm{kg} /$ day.

Children treated as outpatients should be re-evaluated every 1-2 weeks, or sooner, if complications arise or the child's condition is worsening. Treatment should continue until they have no more oedema or until WHZ $>-2$ or until MUAC $>125 \mathrm{~mm}$,

Table 1 Nutrient composition of key therapeutic foods used in the treatment of severe acute malnutrition

\begin{tabular}{llll}
\hline & $\begin{array}{l}\text { F-75 } \\
(100 \mathrm{~mL})\end{array}$ & $\begin{array}{l}\text { F-100 } \\
(100 \mathrm{~mL})\end{array}$ & $\begin{array}{l}\text { Ready-to-use } \\
\text { therapeutic food } \\
(100 \mathrm{~g})\end{array}$ \\
\hline Energy (kcal) & 75 & 100 & 543 \\
Protein (g) & 0.9 & 2.9 & 13.6 \\
Lactose (g) & 1.3 & 4.2 & \\
Potassium (mg) & 156 & 246 & 1111 \\
Sodium (mg) & 14 & 44 & 189 \\
Magnesium (mg) & 10.5 & 17.7 & 92 \\
Zinc (mg) & 2 & 2.3 & 14 \\
Copper (mg) & 0.25 & 0.25 & 1.78 \\
Osmolarity (m0sm/L) & 413 & 419 & \\
\% of total energy from protein & 5 & 12 & $10-12$ \\
\% of total energy from fat & 36 & 53 & $45-60$ \\
\hline
\end{tabular}

depending on which enrolment criteria were used. ${ }^{32}$ In our experience, most children recover within 10-12 weeks and about half recover within 6 weeks if there are no social or compliance concerns and no acute infections that complicate their care. Caretakers of children who do not improve at each visit should be counselled about proper feeding techniques and be queried about whether any acute infections have developed as these children may need additional medical interventions, sometimes including hospitalisation. Repeating the appetite test and having a low threshold for HIV testing in high-prevalence areas are often helpful.

A number of other additional interventions, such as vitamin A and/or folic acid supplementation, antihelminthic medications such as albendazole or mebendazole, antimalarial medication, measles vaccination and routine antibiotics, are variably included in a number of CMAM regimens. However, among these and despite preliminary concerns that they were not helpful, ${ }^{33}$ the routine provision of a short course of oral antibiotics is the only additional intervention to RUTF that has actually been shown to specifically improve recovery rates in this context. ${ }^{12}$

Inpatient therapy consists of a coordinated 10-step protocol (figure 3) whose core element is slow, cautious feeding with F-75 formula every few hours, progressing to F-100 formula as recovery proceeds and when they are able to tolerate this increased solute load. ${ }^{26}$ Additional elements of inpatient therapy address hypoglycaemia, hypothermia, dehydration, electrolyte imbalances and micronutrient deficiencies, although 
Figure 3 Ten-step inpatient management protocol for severe acute malnutrition. Adapted from 2003 WHO guideline. $^{26}$

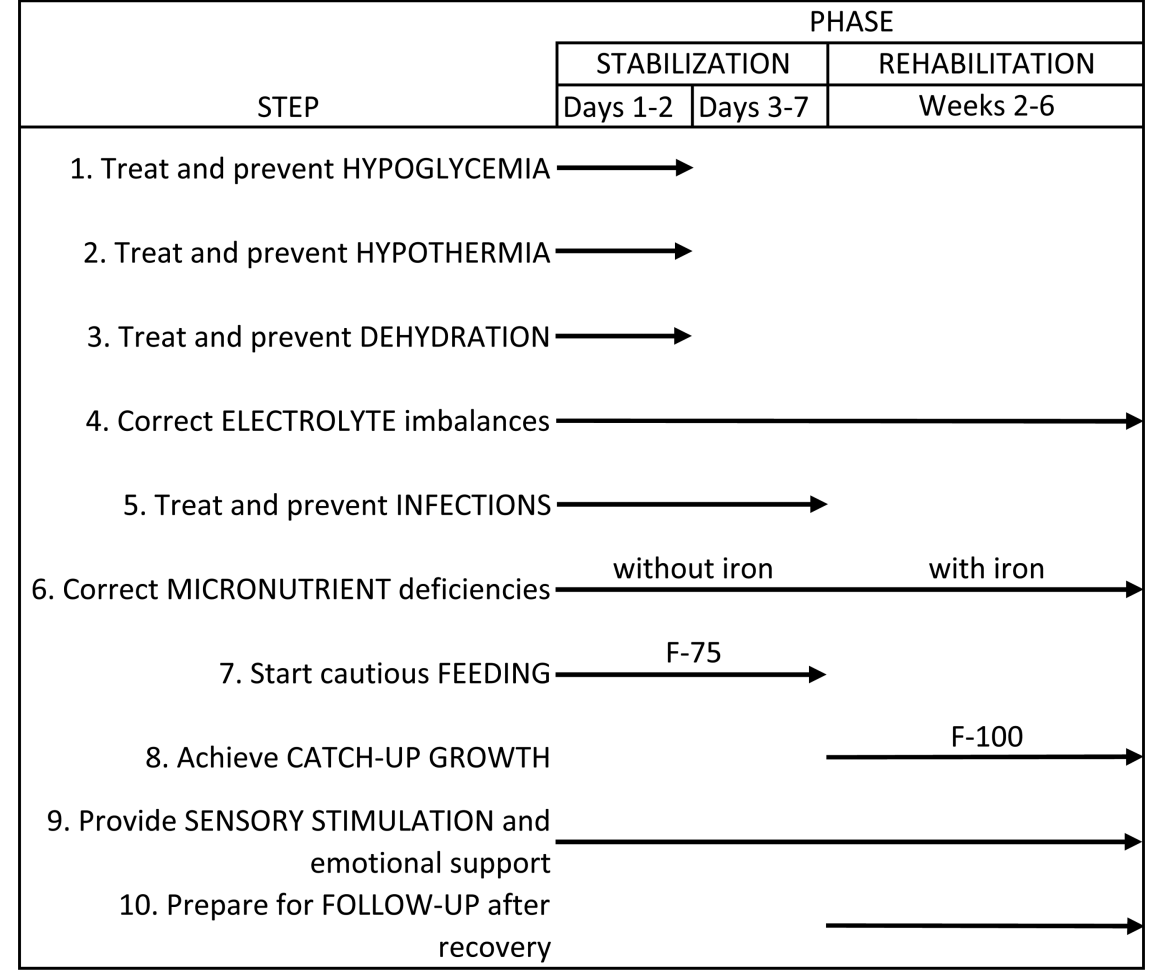

these traditional protocols are being reconsidered in many cases. ${ }^{34}$ Although still an unsettled issue, children with severe dehydration or shock should receive cautious fluid resuscitation as there is at least a strong theoretical risk of fluid overload in these children with compromised cardiac structure and function. ${ }^{35}$ Children treated as inpatients generally receive empiric antibiotic therapy and arrangements should be made to provide developmentally appropriate sensory stimulation and plan for a phased period of follow-up after initial recovery, which may include supplementary food rations after discharge.

\section{PROGNOSIS}

While recovery rates from outpatient therapeutic programmes are generally as good as or better than inpatient programmes, ${ }^{36-}$ 38 there have, in fact, not been the most stringent prospective blinded randomised controlled trials that directly test the efficacy of RUTF. ${ }^{39}$ Nevertheless, more than a decade of operational clinical experience with millions of children has rightly made outpatient management of uncomplicated SAM the de facto standard of care, ${ }^{32}$ and it is unlikely that such a trial could now ethically be conducted.

In the best circumstances, some $90 \%$ of children can be expected to recover from SAM and $<5 \%$ mortality should be achievable. ${ }^{12}$ Untreated episodes of severe wasting typically have a mortality rate of $10 \%-15 \%$ per month; while many will recover spontaneously, their cognitive and physical development likely remains stunted. However, the success of current therapies for SAM underestimates overall mortality as those who recover are at continued risk for relapse and death in the long term, especially those who are HIV-infected. ${ }^{40}$

Further work needs to be done to improve surveillance and case-finding of children with SAM and additional efforts need to be made to treat SAM as a medical emergency, integrating it into the routine delivery of healthcare. This is particularly important in the context of linkage to HIV testing and treatment efforts. ${ }^{27}$
Competing interests None.

Patient consent Obtained.

Provenance and peer review Commissioned; externally peer reviewed.

\section{REFERENCES}

1 Ayele B, Aemere A, Gebre T, et al. Reliability of measurements performed by community-drawn anthropometrists from rural Ethiopia. PLOS ONE 2012;7:e30345.

2 Wootton S, Durkin K, Jackson A. Quality control issues related to assessment of body composition. Food Nutr Bull 2014;35:S79-85.

3 Cogill B. Anthropometric indicators measurement guide. Washington, DC: Food and Nutrition Technical Assistance Project, Academy for Educational Development, 2003.

4 Mogeni P, Twahir $H$, Bandika V, et al. Diagnostic performance of visible severe wasting for identifying severe acute malnutrition in children admitted to hospital in Kenya. Bull World Health Organ 2011;89:900-6.

5 WHO. WHO child growth standards and the identification of severe acute malnutrition in infants and children. Geneva: World Health Organization, United Nations Children's Fund, 2009.

6 WHO. WHO Child Growth Standards. http://www.who.int/childgrowth (accessed 24 Sep 2014).

7 Briend A, Maire B, Fontaine 0 , et al. Mid-upper arm circumference and weight-for-height to identify high-risk malnourished under-five children. Matern Child Nutr 2012:8:130-3.

8 Myatt M, Khara T, Collins S. A review of methods to detect cases of severely malnourished children in the community for their admission into community-based therapeutic care programs. Food Nutr Bull 2006;27:57-23.

9 Ahmed T, Rahman S, Cravioto A. Oedematous malnutrition. Indian J Med Res 2009;130:651-4.

10 Heikens GT, Manary M. 75 years of Kwashiorkor in Africa. Malawi Med J 2009:21:96-8.

11 Heilskov S, Rytter MJ, Vestergaard C, et al. Dermatosis in children with oedematous malnutrition (kwashiorkor): a review of the literature. J Eur Acad Dermatol Venereol 2014;28:995-1001.

12 Trehan I, Goldbach HS, LaGrone LN, et al. Antibiotics as part of the management of severe acute malnutrition. N Engl J Med 2013;368:425-35.

13 Smith MI, Yatsunenko T, Manary MJ, et al. Gut microbiomes of Malawian twin pairs discordant for kwashiorkor. Science 2013;339:548-54.

14 Subramanian S, Huq S, Yatsunenko T, et al. Persistent gut microbiota immaturity in malnourished Bangladeshi children. Nature 2014;510:417-21.

15 WHO. Emergency Triage Assessment and Treatment (ETAT) Course. http://www. who.int/maternal_child_adolescent/documents/9241546875/en/ (accessed 24 Sep 2014) 
16 WHO. WHO/UNICEF joint statement: integrated Community Case Management (iCCM): an equity-focused strategy to improve access to essential treatment services for children. New York: World Health Organization, United Nations Children's Fund 2012.

17 Black RE, Victora CG, Walker SP, et al. Maternal and child undernutrition and overweight in low-income and middle-income countries. Lancet 2013;382:427-51.

18 Ahmed T, Hossain M, Mahfuz M, et al. Severe acute malnutrition in Asia. Food Nutr Bull 2014;35:S14-26.

19 Briend $A$, Collins S, Golden $M$, et al. The burden of severe acute malnutrition estimated in the Lancet series is a minimum rather than an actual estimate. Lancet 2013;382:1549.

20 Bahwere P. Severe acute malnutrition during emergencies: burden management, and gaps. Food Nutr Bull 2014;35:S47-51.

21 Gross R, Webb P. Wasting time for wasted children: severe child undernutrition must be resolved in non-emergency settings. Lancet 2006;367:1209-11.

22 Uauy R, Desjeux JF, Ahmed T, et al. Global efforts to address severe acute malnutrition. J Pediatr Gastroenterol Nutr 2012;55:476-81.

23 Bhutta ZA, Das JK, Rizvi A, et al. Evidence-based interventions for improvement of maternal and child nutrition: what can be done and at what cost? Lancet 2013;382:452-77.

24 DeBoer MD, Lima AA, Oria RB, et al. Early childhood growth failure and the developmental origins of adult disease: do enteric infections and malnutrition increase risk for the metabolic syndrome? Nutr Rev 2012;70:642-53.

25 Uauy R, Garmendia ML, Corvalan C. Addressing the double burden of malnutrition with a common agenda. Nestle Nutr Inst Workshop Ser 2014;78:39-52.

26 Ashworth A, Khanum S, Jackson A, et al. Guidelines for the inpatient treatment of severely malnourished children. Geneva: World Health Organization, 2003.

27 Trehan I, O'Hare BA, Phiri A, et al. Challenges in the management of HIV-infected malnourished children in Sub-Saharan Africa. AIDS Res Treat 2012;2012:790786.

$28 \mathrm{Kim} \mathrm{MH}, \mathrm{Cox}$ C, Dave A, et al. Prompt initiation of ART with therapeutic food is associated with improved outcomes in HIV infected Malawian children with malnutrition. J Acquir Immune Defic Syndr 2012;59:173-6.

29 Murray E, Manary M. Home-based therapy for severe acute malnutrition with ready-to-use food. Paediatr Int Child Health 2014. [Epub ahead of print].
30 WHO. Community-based management of severe acute malnutrition. World Health Organization, World Food Programme, United Nations System Standing Committee on Nutrition, United Nations Children's Fund, 2007.

31 Santini A, Novellino E, Armini V, et al. State of the art of Ready-to-Use Therapeutic Food: a tool for nutraceuticals addition to foodstuff. Food Chem 2013;140:843-9.

32 WHO. Guideline: updates on the management of severe acute malnutrition in infants and children. Geneva: World Health Organization, 2013.

33 Trehan I, Amthor RE, Maleta K, et al. Evaluation of the routine use of amoxicillin as part of the home-based treatment of severe acute malnutrition. Trop Med Int Health 2010;15:1022-8.

34 Brewster DR. Inpatient management of severe malnutrition: time for a change in protocol and practice. Ann Trop Paediatr 2011;31:97-107.

35 Obonyo N, Maitland K. Fluid management of shock in severe malnutrition: what is the evidence for current guidelines and what lessons have been learned from clinical studies and trials in other pediatric populations? Food Nutr Bull 2014;35:S71-8.

36 Diop el HI, Dossou NI, Ndour MM, et al. Comparison of the efficacy of a solid ready-to-use food and a liquid, milk-based diet for the rehabilitation of severely malnourished children: a randomized trial. Am J Clin Nutr 2003;78:302-7.

37 Ciliberto MA, Sandige H, Ndekha MJ, et al. Comparison of home-based therapy with ready-to-use therapeutic food with standard therapy in the treatment of malnourished Malawian children: a controlled, clinical effectiveness trial. Am J Clin Nutr 2005;81:864-70.

38 Bahwere $\mathrm{P}$, Mtimuni A, Sadler K, et al. Long term mortality after community and facility based treatment of severe acute malnutrition: Analysis of data from Bangladesh, Kenya, Malawi and Niger. J Pub Health Epid 2012;4:215-25.

39 Schoonees A, Lombard M, Musekiwa A, et al. Ready-to-use therapeutic food for home-based treatment of severe acute malnutrition in children from six months to five years of age. Cochrane Database Syst Rev 2013;6:CD009000.

40 Kerac M, Bunn J, Chagaluka G, et al. Follow-up of post-discharge growth and mortality after treatment for severe acute malnutrition (FuSAM study): a prospective cohort study. PLoS ONE 2014;9:e96030.

41 Manary MJ, Sandige HL. Management of acute moderate and severe childhood malnutrition. BMJ 2008;337:a2180. 